Revista PSICOLOGIA, 2015, Vol. 29 (2), 47-62. doi: http://dx.doi.org/10.17575/rpsicol.v29i2.1031

\title{
A prevalência do Transtorno do Déficit de Atenção e Hiperatividade (TDAH): uma revisão de literatura
}

\author{
Ana Flávia Hora ${ }^{1}$, Simone Silva ${ }^{1}$, Maely Ramos ${ }^{1}$, Fernando Pontes ${ }^{1}$, João Paulo Nobre ${ }^{1}$ \\ ${ }^{1}$ Universidade Federal do Pará, Brasil
}

\begin{abstract}
Resumo: Este artigo expõe as principais características metodológicas e os resultados apresentados pelos estudos de prevalência do Transtorno do Déficit de Atenção e Hiperatividade (TDAH) através de artigos publicados no período de 2003 a 2014. Realizou-se uma busca em uma das principais bases de dados da área da saúde, o PubMed/MEDLINE que resultou em 23 artigos. Investigou-se as estimativas de prevalência; o processo de amostragem; o tipo de estudo; os critérios diagnósticos; e, as principais variáveis exploradas. 0 processo de mineração foi realizado pelos programas: EXCEL; NVIVO-10, SPSS 20; e MENDELEY. Resultados: Observou-se a média central de $11.26 \%$ entre as estimativas de prevalência encontradas; $99 \%$ dos estudos foram transversais; e o processo de amostragem mais utilizado foi o probabilístico por conveniência em $26.08 \%$ dos estudos. Os resultados alcançados apoiam a premissa de que os critérios metodológicos e diagnósticos ampliam a variabilidade das estimativas de prevalência a nível global.
\end{abstract}

Palavras-chave: Prevalência; TDAH; Epidemiologia; Crianças; Adolescentes.

The prevalence of ADHD: a literature review: This article presents the main methodological characteristics and the results reported by the studies of the prevalence of ADHD through the articles published in the 2003-2014. We performed a search on one of the major health care databases, PubMed / MEDLINE which resulted in 23 articles. We investigated the prevalence estimates; the sampling process; the type of study; the diagnostic criteria; and the main variables explored. The mining process was carried out by the programs: EXCEL; NVivo-10, SPSS 20; and Mendeley. Results: We observed the central average of $11.26 \%$ and among estimates of prevalence found; $99 \%$ of the studies were cross-sectional; and the most widely used sampling process was the probabilistic for convenience in $26.08 \%$ of the studies. Conclusion: The results support the premise that the methodological criteria and diagnoses, increased the variability of estimates of prevalence globally.

Keywords: Prevalence; ADHD; Epidemiology; Children; Adolescents.

A prevalência do Transtorno do Déficit de Atenção e Hiperatividade (TDAH) tem sido objeto de estudo de diversas investigações científicas nas últimas décadas, tais estudos têm proporcionado dados sobre a frequência, severidade e curso do transtorno em relação ao gênero, idade, área geográfica, fatores psicossociais, etc. (Díaz, Jiménez, Rodríguez, \& Afonso, 2013; Faraone, Sergeant, Gillberg, \& Biederman, 2003; Hodgkins et al., 2012; Polanczyk et al., 2014).

O TDAH caracteriza-se pela tríade sintomatológica de desatenção, hiperatividade e impulsividade, que se manifestam de maneira desproporcional tendo em vista a idade e o nível de desenvolvimento do indivíduo (Cornejo et al., 2005; Rohde et al., 2005; Sánchez, Velarde, \& Britton, 2011). 0 transtorno é considerado pela Associação Americana de Psiquiatria (American Psychiatric Association, 2013) como um problema de saúde pública cujas implicações consistem em atividades motoras excessivas, na dificuldade em sustentar a atenção e, no controle dos impulsos. Estas características podem comprometer o comportamento funcional do indivíduo no âmbito familiar, social, laboral e, acadêmico (Jin, Du, Zhong, \& David, 2013).

As crianças, adolescentes e adultos com o transtorno estão sujeitos a apresentar problemas na esfera social, interpessoal e intrapessoal, tais como: baixa autoestima, conflitos familiares, problemas de relacionamento entre iguais e conjugais (Biederman et al., 2012; Vaquerizo-Madrid, 2005), maior probabilidade de envolvimento em acidentes automobilísticos, práticas sexuais de risco, uso de substâncias ilícitas, comportamentos antissociais, entre outros (Barkley \& Cox, 2007; Froehlich et al., 2007; Polanczyk et al., 2014; Ranby et al., 2012).

\footnotetext{
${ }^{1}$ Dados de contacto para correspondência: Ana Flávia da Hora, Rua Bahia, Cond. Gran Village São Luís, Bl 14, Ap. 103, Cep: 65065720, Brasil. E-mail: anaflaviadahora@hotmail.com.
} 
Mesmo com os avanços dos estudos genéticos e das técnicas de neuroimagem, ainda não há um consenso definitivo sobre a etiologia do transtorno. A literatura científica sinaliza que é uma síndrome heterogênea de origem multifatorial, integrando fatores genéticos, neurobiológicos, ambientais e múltiplos genes associados (Biederman, 2005; Dye, 2013; McGough \& McCracken, 2006; Polanczyk et al., 2014; Ranby et al., 2012).

Como não há marcadores biológicos específicos, o diagnóstico do transtorno é essencialmente clínico, a partir da observação da manifestação dos sintomas listados nos sistemas de classificação de saúde: o DSM - Manual Diagnóstico e Estatísticos de Transtorno Mental e o CID - Classificação Internacional de Doenças (Cardo \& Servera, 2008; Jara, 2009). Devido às suas características clínicas, é um dos transtornos crônicos mais frequentes em crianças e adolescentes encaminhados aos serviços ambulatoriais especializados em saúde mental (Catalá-López et al., 2012; Ndukuba, Odinka, Muomah, Obindo, \& Omigbodun, 2014; Ramtekkar, Reiersen, Todorov, \& Todd, 2010).

Diante da gravidade do transtorno, convém destacar que os estudos de prevalência são imprescindíveis no monitoramento da frequência do TDAH na população, e na identificação dos fatores de risco e consequências na saúde pública (Catalá-López et al., 2012; Emerich, da Rocha, Silvares, \& Gonçalves, 2012; Fernández, 2005). Tais estudos auxiliam na melhoria da qualidade de vida do indivíduo por identificar medidas preventivas e possíveis formas de controle (Alvarez \& Claros, 2012; HernándezAvila, Garrido-Latorre, \& López-Moreno, 2000).

Para os órgãos governamentais que elaboram e planejam as políticas de assistência à saúde, tornase fundamental o conhecimento da prevalência de uma determinada patologia na população para a alocação e priorização de recursos destinados à prevenção, proteção e promoção da saúde (Díaz et al., 2013; Fernández, 2005; Hernández-Avila et al., 2000). Segundo o relatório da Organização Mundial de Saúde (Dye, 2013), as investigações científicas têm sido indispensáveis no desenvolvimento da saúde pública, pois além de investigarem a distribuição da enfermidade na população, possibilitam a análise dos possíveis fatores determinantes (Catalá-López et al., 2012; Díaz et al., 2013; Hernández-Avila et al., 2000).

Apesar das contribuições dos estudos de prevalência, é possível identificar algumas limitações, visto que, na qualidade de descritivos populacionais, estão condicionados aos critérios metodológicos, à seleção da amostra representativa da população-alvo, à localização geográfica, à precisão diagnóstica, aos fatores sociodemográficos, e à fonte de informações utilizadas como forma de rastreamento (Froehlich et al., 2007; López-Soler, Sáez, López, Fernández, \& Pina, 2009; Ndukuba et al., 2014).

Reconhecendo a importância da análise dos estudos de prevalência do TDAH, o presente estudo de revisão objetivou: a) construir um panorama dos estudos de prevalência do TDAH em crianças e adolescentes na faixa etária dos 03 aos 17 anos b) Rastrear artigos publicados no período de 2003 a 2014; c) identificar as prevalências estimadas pelos diferentes estudos a nível internacional d) realizar uma análise descritiva das características metodológicas, das variáveis exploradas, e dos critérios de diagnóstico adotados nas pesquisas realizadas no período estabelecido.

\section{MÉTODO}

\section{Estratégias de Busca e Codificação dos Artigos}

Realizou-se um levantamento dos estudos epidemiológicos do TDAH seguindo as diretrizes da Medicina Baseada em Evidências (BEM) com base nas orientações da Colaboração Cochrane para as revisões sistemáticas (Galvão, Sawada, \& Trevizan, 2004).

Esta revisão foi constituída por seis etapas: (1) formulação da pergunta/problema; (2) localização e seleção dos estudos; (3) avaliação crítica dos estudos; (4) coleta de dados nos artigos; (5) análise e apresentação dos dados; (6) interpretação dos dados (Cordeiro, Oliveira, Rentería, \& Guimarães, 2007).

$\mathrm{Na}$ primeira etapa, formulou-se o problema a partir da seguinte questão: Quais as principais características metodológicas dos estudos de prevalência do TDAH em crianças e adolescentes em contexto escolar? Considerando-se a estrutura P.V.O: P (situação problema, participantes ou contexto) crianças ou adolescentes no contexto escola; V (variável principal do estudo) - a prevalência do TDAH; 0 (resultado esperado) - identificar as principais características metodológicas dos estudos sobre a temática em questão.

$\mathrm{Na}$ segunda etapa (localização e seleção dos artigos), realizou-se uma busca na base PubMed/MEDLINE por ser uma das bases de dados mais utilizadas pelos profissionais da área da saúde, com base no DeCS (Descritores em Ciências da Saúde) e no P.V.O, definindo-se, assim, os seguintes termos: TDAH, “ADHD”, epidemiologia, "epidemiology”, “ epidemiología”, prevalência, "prevalence”, e "prevalencia".

Com a escolha dos descritores, delimitam-se as estratégias de busca na base de dados através da aplicação de operadores booleanos aos componentes da escala P.V.O. Desta maneira, para o levantamento 
de artigos a partir de uma estratégia em inglês, traduziram-se para o português e o espanhol os termos equivalentes, a saber:

("adhd" OR "epidemiology adhd" OR "prevalence adhd") AND "epidemiology" AND "prevalence" ("tdah" OR "epidemiologia tdah" OR "prevalência tdah") AND "epidemiologia" AND "prevalência". ("tdah" OR “epidemiología tdah" OR "prevalencia tdah") AND “epidemiología” AND "prevalencia”.

Na terceira etapa, para avaliação dos artigos levantados foram considerados os seguintes critérios de inclusão: a) estudos de delineamento transversal ou longitudinal; b) artigos que apresentavam a estimativa de prevalência do TDAH; c) estudos compostos por amostras de crianças e adolescentes dos 3 aos 17 anos; d) artigos originais e publicados na sua totalidade; e) estudos que descrevessem os instrumentos utilizados na seleção da amostra; e f) artigos que apresentavam os descritores de busca no título. E como critérios de exclusão: a) artigos repetidos; b) artigos com amostras de adultos; c) estudos que não estimavam a prevalência do TDAH d) estudos que estimavam a prevalência dos transtornos comórbidos; e) estudos que não descreviam os instrumentos utilizados.

Para a avaliação crítica dos estudos, elaboraram-se dois testes de relevância para avaliadores distintos com o objetivo de aumentar a confiabilidade dos resultados. 0 Teste de Relevância I foi aplicado aos artigos por um avaliador com base nas seguintes questões: 0 estudo é sobre prevalência do TDAH? 0 objetivo do estudo está apresentado claramente? O estudo tem acesso livre aos pesquisadores? Os participantes do estudo eram crianças ou adolescentes? As respostas podiam ser negativas ou positivas.

No Teste de Relevância II foram considerados apenas os artigos aprovados pelo Teste de Relevância I e foram aplicados por dois juízes independentes a partir das seguintes questões: 1aㅡ 0 estudo apresenta claramente o processo de seleção da amostra?; $2^{a}$ ) Os instrumentos utilizados são compatíveis com os objetivos estabelecidos?; 3aㅡ A análise estatística da estimativa de prevalência está descrita?; 4무 0 estudo apresenta as taxas de prevalência encontradas; e a $5^{\underline{a}}$ ) Os resultados estão descritos? Após a análise dos juízes, foram considerados os artigos que possuíam um índice de concordância IC $>80 \%$. O IC foi calculado a partir da fórmula: $\mathrm{IC}=\mathrm{A} /(\mathrm{A}+\mathrm{D}) \times \mathrm{x} 100$, cuja legenda se discrimina da seguinte maneira: $\mathrm{IC}$ = índice de concordância; $\mathrm{A}=$ concordância; $\mathrm{D}=$ discordância.

Na quarta etapa (coleta de dados), analisou-se a caracterização geral dos estudos epidemiológicos do TDAH, explorando: a) Prevalência do TDAH; b) Aspectos metodológicos c) Resultados dos estudos variáveis exploradas.

$\mathrm{Na}$ análise dos dados (5⿳亠丷厂 etapa), optou-se pela seleção dos principais achados utilizando-se os seguintes programas: (1) EXCEL para a elaboração de planilhas e tabulação dos dados; (2) NVIVO-10 para a análise de conteúdo dos artigos; (3) SPSS 20 para a realização da análise estatística central da amostra sua totalidade através da estatística descritiva (média, desvio-padrão, valores mínimos e máximos) e; (4) MENDELEY para a organização das referências.

Convém explicitar que um grafo é formado por vértices (pontos/elemento) e arestas (ligações entre pontos - conexões) que representam associações entre elementos (Guizzo et al., 2003; Lage, 2011). Os grafos possuem métricas (medidas) específicas que contribuem na investigação entre os elementos dos estudos, por isso optou-se por essa técnica para a análise das conexões entre as palavras-chave dos artigos. As informações coletadas foram interpretadas após a organização e análise de dados (etapa 6).

\section{RESULTADOS}

\section{Análise de Dados}

O levantamento inicial resultou em 141 artigos que foram obtidos através dos descritores e cruzamentos pré-determinados. Sequencialmente, dois testes de relevância foram aplicados para verificar a adequabilidade dos estudos aos critérios pré- estabelecidos. Os resultados sinalizam que no refinamento inicial $12 \%$ dos artigos foram excluídos por não terem sido revisados por pares e $67 \%$ por serem repetidos, totalizando o quantitativo de 30 artigos.

Na aplicação do Teste de Relevância I, três artigos aprovados inicialmente foram excluídos, pois dois não identificavam os instrumentos utilizados na coleta de dados e, 1 artigo não descrevia claramente os respondentes dos instrumentos de avaliação do TDAH. Por sua vez, no Teste de relevância II, quatro artigos tiveram um IC $<80 \%$ de concordância entre os juízes, os quais foram submetidos a nova avaliação em que se optou por excluí-los, ou seja, dos 30 artigos submetidos aos testes, 7 foram excluídos, totalizando um quantitativo de 23 artigos (11 asiáticos, 7 americanos, 3 europeus, e 2 africanos), isto é, apenas $16 \%$ se adaptaram ao modelo de exigência adotado.

\section{Estimativas de Prevalência}

Em relação às taxas de prevalência encontradas nos diferentes países, observaram-se as estimativas que variaram respectivamente de $2.7 \%$ a $31.1 \%$, como também, a faixa etária dos sujeitos participantes dos 3 aos 17 anos, conforme se verifica no Quadro 1. 
Quadro 1. Prevalência dos estudos.

\begin{tabular}{lll}
\hline País & Prevalência & Idade \\
\hline Arábia Saudita (Alqahtani, 2010) & $2.7 \%$ & $7-9$ anos \\
China (Jin, Du, Zhong, \& David, 2013) & $4.6 \%$ & $5-15$ anos \\
Espanha (Cardo, Servera, \& Llovera, 2007) & $4.6 \%$ & $6-11$ anos \\
Noruega (Ullebø, Posserud, Heiervang, Obel, \& Gillberg, 2012) & $5.2 \%$ anos \\
República do Congo (Kashala, Tylleskar, Elgen, Kayembe, \& Sommerfelt, 2005) & $6 \%$ & $7-9$ anos \\
Nigéria (Ndukuba, Odinka, Muomah, Obindo, \& Omigbodun, 2014) & $6.6 \%$ & $6-8$ anos \\
Brasil (Freire \& Pondé, 2005) & $6.7 \%$ & $6-17$ anos \\
Panamá (Sánchez, Velarde, \& Britton, 2011) & $7.4 \%$ & $6-11$ anos \\
Inglaterra (Alloway, Elliott, \& Holmes, 2010) & $8 \%$ & 10 anos \\
Estados Unidos da América (Wolraich et al., 2012) & $8.7 \% ; 10.6 \%$ & $5-13$ anos \\
Catar (Bener, Qahtani, \& Abdelaal, 2006) & $9.4 \%$ & $6-12$ anos \\
Irão (Amiri, Fakhari, Maheri, \& Mohammadpoor Asl, 2010) & $9.7 \%$ & $7-15$ anos \\
Venezuela (Montiel-Nava, Peña, \& Montiel-Barbero, 2003) & $10.15 \%$ & $3-13$ anos \\
Catar (Bener, Al Qahtani, Teebi, \& Bessisso, 2008) & $11.1 \%$ & $6-12$ anos \\
Espanha (Sánchez, Ramos, \& Díaz, 2014) & $11.52 \%$ & $6-16$ anos \\
Índia (Ajinkya, Kaur, Gursale, \& Jadhav, 2012) & $12.3 \%$ & $6-13$ anos \\
Irão (Abdekhodaie, Tabatabaei, \& Gholizadeh, 2012) & $12.3 \%$ & $5-6$ anos \\
Brasil (Fontana, De Vasconcelos, Werner, De Góes, \& Liberal, 2007) & $13 \%$ & $6-12$ anos \\
Irão (Talaei, Mokhber, Abdollahian, Bordbar, \& Salari, 2010) & $15.27 \%$ & $7-9$ anos \\
Brasil (Vasconcelos et al., 2003) & $17.1 \%$ & $6-12$ anos \\
Colômbia (Cornejo et al., 2005) & $20.4 \%$ & $4-17$ anos \\
Irão (Meysamie, Fard, \& Mohammadi, 2011) & $25.8 \%$ & $3-6$ anos \\
Japão (Soma, Nakamura, Oyama, Tsuchiya, \& Yamamoto, 2009) & $31.1 \%$ & $3-6$ anos \\
\hline
\end{tabular}

Encontrou-se uma média de $11.26 \%$ (95\% IC= $8.4-14.1)$ através do teste student $\mathrm{t}(p<0.001)$. Comparando os diferentes grupos de faixa etária, observaram-se maiores estimativas de prevalência em amostras de pré-escolares de 3 a 6 anos apresentando médias de 25.8\% (Meysamie, Fard, \& Mohammadi, 2011) e 31.1\% respectivamente (Soma, Nakamura, Oyama, Tsuchiya, \& Yamamoto, 2009).

Convém ressaltar as estimativas coincidentes em países distintos com diferentes amostras, como 4.6\% na China encontrada na cidade de Xangai com uma amostra de 5648 estudantes de 5 a 15 anos (Jin et al., 2013), e nas Ilhas Canárias da Espanha com 1509 estudantes na faixa etária de 6 a 11 anos (Servera \& Cardo, 2007). Como também, a taxa de 12.3\% encontrada em uma amostra de 1083 estudantes de 5 a 6 anos, na pesquisa realizada em 10 áreas urbanas do nordeste do Irão (Abdekhodaie, Tabatabaei, \& Gholizadeh, 2012), e na Índia na cidade de Navi Mumbai com 132 estudantes (Ajinkya, Kaur, Gursale, \& Jadhav, 2012).

Algumas estimativas de prevalência foram relativamente próximas com amostras também distintas em proporção, como o estudo realizado na Nigéria com 200 estudantes de 6 a 8 anos que, apontou a prevalência de 6.6\% (Ndukuba et al., 2014) e, a investigação efetuada no Brasil com 774 estudantes de 6 a 17 anos com a estimativa de 7.6\% (Freire \& Pondé, 2005). De igual modo, o estudo realizado no Qatar com 1541 estudantes de 6 a 12 anos, estimou a prevalência de 9.4\% (Bener, Qahtani, \& Abdelaal, 2006), e a pesquisa administrada no Irão (Amiri, Fakhari, Maheri, \& Mohammadpoor Asl, 2010) com 1658 estudantes de 7 a 15 anos, sinalizou uma taxa de prevalência de 9.7\%.

\section{Aspectos Metodológicos}

Compreendendo que os estudos epidemiológicos são importantes por proporcionarem a identificação da incidência do transtorno em uma amostra representativa da população, torna-se imprescindível analisar os procedimentos metodológicos utilizados pelas pesquisas. Deste modo, a seguir apresentam-se os tipos de estudos e o processo de amostragem encontrados na presente revisão (Figura 1). 


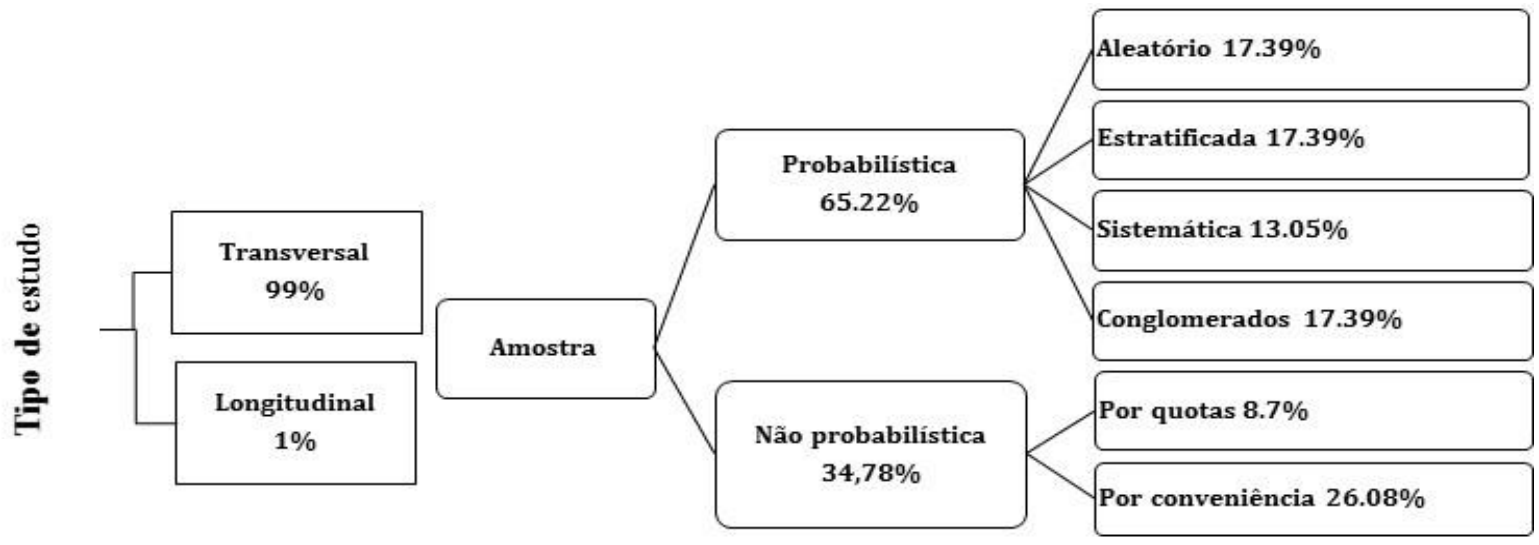

Figura 1. Diagrama dos aspectos metodológicos

Dos artigos categorizados, 99\% foram do tipo transversal, avaliando o fenômeno do TDAH e as variáveis em um único momento, enquanto que 1\% foi do tipo longitudinal (Soma et al., 2009) em que se avaliou a evolução dos sintomas do TDAH em pré-escolares através do relato dos professores em 2003 e dos pais em 2006.

Por conseguinte, analisou-se a seleção da amostra (Figura 1), pois compreende-se que esta serve de base para as generalizações dos dados em uma dada população. Esquematizaram-se os tipos de amostra em duas categorias: probabilística, em que todos os indivíduos de uma população tiveram a mesma probabilidade de serem selecionados, e não probabilística, em que nem todos os indivíduos de uma população poderiam ser escolhidos (Szwarcwald \& Damacena, 2008).

Do método probabilístico, $17.39 \%$ dos pesquisadores utilizaram a seleção amostral de maneira aleatória simples (Alqahtani, 2010; Bener, Al Qahtani, Teebi, \& Bessisso, 2008; Freire \& Pondé, 2005; Sánchez et al., 2011) por meio de sorteio randômico dos sujeitos nas escolas onde todos tinham igual probabilidade de serem escolhidos. Outro tipo de amostra foi a estratificada, em que $17.39 \%$ dos estudos (Fontana, De Vasconcelos, Werner, De Góes, \& Liberal, 2007; Montiel-Nava, Peña, \& Montiel-Barbero, 2003; Sánchez, Ramos, \& Díaz, 2014) dividiram a população em estratos, e depois realizaram a seleção.

Por sua vez, 13.05\% dos estudos (Bener et al., 2006; Cornejo et al., 2005; Wolraich et al., 2012) selecionaram a amostra de maneira sistemática cujo critério de probabilidade só ocorreu depois da seleção aleatória do primeiro grupo amostral. Ademais, 17.39\% (Abdekhodaie et al., 2012; Amiri et al., 2010; Kashala, Tylleskar, Elgen, Kayembe, \& Sommerfelt, 2005; Meysamie et al., 2011) usaram a estratégia de seleção por conglomerados cuja população de escolares foi dividida em grupos, e depois foram avaliados com instrumentos psicométricos específicos para a definição da amostra final.

Do tipo de amostragem não probabilística, 8.70\% dos estudos utilizaram o método de seleção por quotas (Alloway, Elliott, \& Holmes, 2010; Soma et al., 2009) estruturada por grupo e selecionada a partir de critérios específicos, como subtipos do TDAH, gênero, idade, etc. Por outro lado, 26.08\% dos estudos (Ajinkya et al., 2012; Jin et al., 2013; Ndukuba et al., 2014; Talaei, Mokhber, Abdollahian, Bordbar, \& Salari, 2010; Ullebø, Posserud, Heiervang, Obel, \& Gillberg, 2012) selecionaram a amostra por conveniência, conforme retornavam devidamente preenchidos os questionários enviados aos pais.

A análise dos processos metodológicos permitiu identificar os instrumentos utilizados na detecção do TDAH para avaliar os comportamentos do ponto de vista do adolescente, pais e professores. Dos 23 artigos, 11 (Abdekhodaie et al., 2012; Alloway et al., 2010; Amiri et al., 2010; Cornejo et al., 2005; Fontana et al., 2007; Freire \& Pondé, 2005; Jin et al., 2013; Meysamie et al., 2011; Talaei et al., 2010; Vasconcelos et al., 2003; Wolraich et al., 2012) adotaram o DSM-IV para o diagnóstico clínico do TDAH, não obstante, 1 artigo (Soma et al., 2009) fez uso do DSM-III. Por sua vez, dois artigos (Cardo, Servera, \& Llovera, 2007; Ndukuba et al., 2014) utilizaram o ADHD Rating Scale-IV.

Em contrapartida, sete artigos adotaram o Questionário de Conners, sendo que cinco (Amiri et al., 2010; Cornejo et al., 2005; Montiel-Nava et al., 2003; Sánchez et al., 2011; Talaei et al., 2010) usaram a versão para pais e professores, e dois se restringiram a versão para pais (Abdekhodaie et al., 2012; Bener et al., 2006); três estudos utilizaram a Escala Vanderbilt (Ajinkya et al., 2012; Alqahtani, 2010; Wolraich et al., 2012), e dois artigos (Kashala et al., 2005; Wolraich et al., 2012) adotaram o Questionário SDQ (Questionário de Capacidades e dificuldades). Convém destacar que um artigo (Sánchez et al., 2011) fez uso restrito da escala EDAH (Avaliação do Transtorno de déficit de atenção e hiperatividade) para professores; e um artigo (Ullebø et al., 2012) adotou o SNAP-IV.

Em relação aos respondentes dos testes, 17 artigos (Abdekhodaie et al., 2012; Alqahtani, 2010; Amiri et al., 2010; Cornejo et al., 2005; Fontana et al., 2007; Jin et al., 2013; Kashala et al., 2005; Meysamie 
et al., 2011; Montiel-Nava et al., 2003; Ndukuba et al., 2014; Sánchez et al., 2011; Soma et al., 2009; Talaei et al., 2010; Ullebø et al., 2012; Vasconcelos et al., 2003; Wolraich et al., 2012) consideraram os pais e os professores como os principais respondentes dos instrumentos de coleta, ainda que os mesmos não estivessem de acordo em relação à manifestação dos sintomas do transtorno; em cinco artigos (Alloway et al., 2010; Bener et al., 2008; Bener et al., 2006; Freire \& Pondé, 2005) os professores foram os respondentes e um artigo (Ajinkya et al., 2012) restringiu-se à avaliação dos pais.

\section{Resultados dos Estudos - Variáveis Exploradas}

Para a análise de conteúdo dos resultados dos artigos levantados, utilizou-se o software NVIVO 10. Para tanto, foram cumpridas as seguintes etapas: (1) organização e gerenciamento das fontes de dados no software; (2) codificação das fontes; (3) visualização dos resultados de codificação. Na fonte de dados resultante dos artigos selecionados destacaram-se as variáveis investigadas pelos pesquisadores. Após a organização desses dados (etapa 1) realizou-se o processo de codificação (etapa 2) por meio da identificação de Nodes ou Nós.

Os nós são componentes de armazenamento de informações codificadas que indicam as temáticas exploradas nos artigos. Por se tratar de uma análise de conteúdo, os nós (Figura3) receberam os códigos, formando categorias de informações descritivas sobre as fontes de dados em análise (Guizzo et al., 2003; Lage, 2011).

Os nós podem ser organizados de duas formas no software Nvivo 10, a saber: Free Node (em que a codificação é formada por um nó isolado) e; Tree Node (há um nó principal e outros subnós hierarquicamente dependentes). Nesta revisão a estrutura da codificação é Tree Node. Ressalta-se que as categorias não foram pré-definidas, mas emergiram da análise de conteúdo realizada nos resultados dos artigos investigados. Sendo assim, foram criados um nó principal (Epidemiologia do TDAH) e 44 subnós (Figura 2) formando uma árvore de nós (Guizzo et al., 2003).

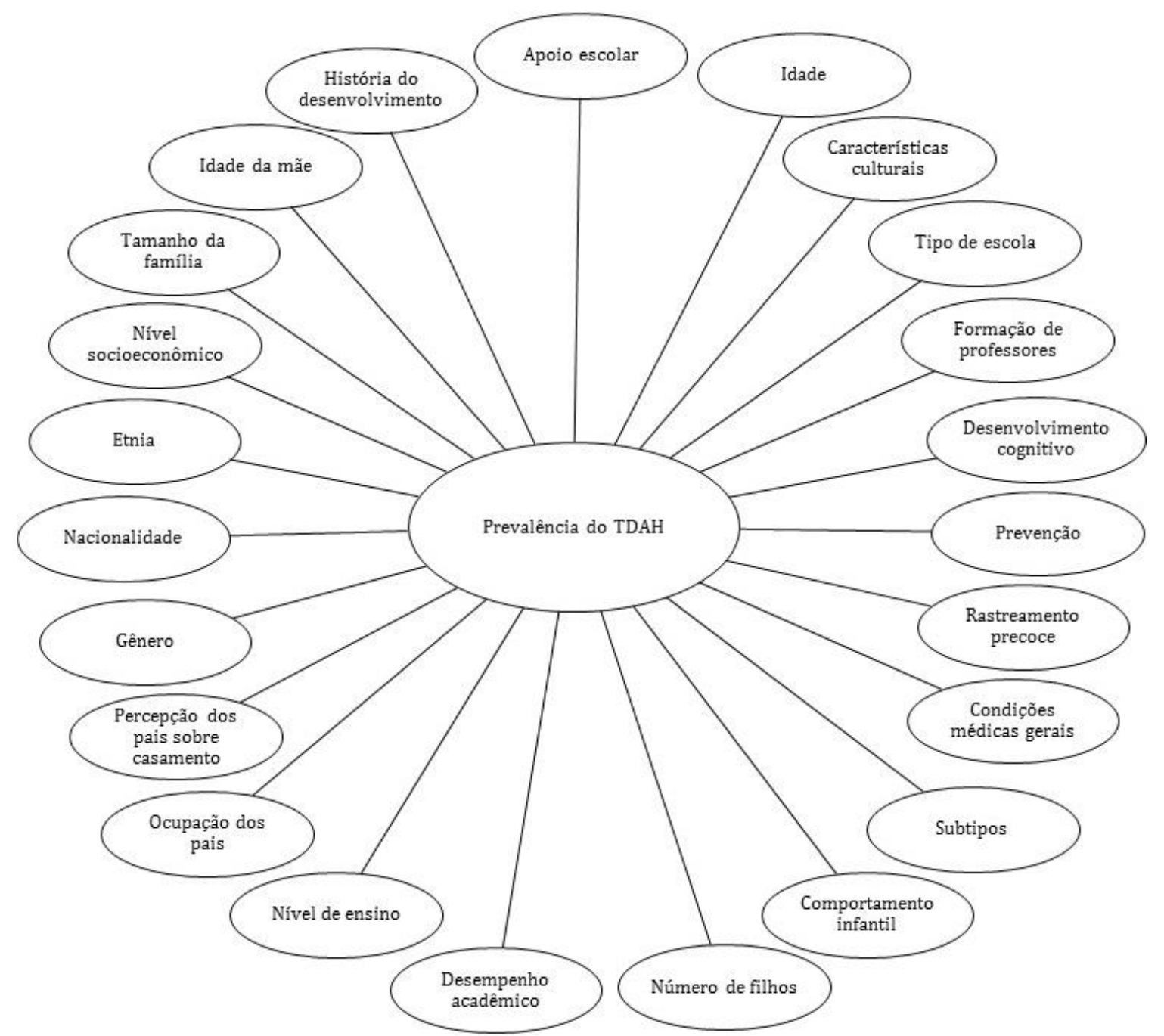

Figura 2. Modelo exploratório das categorias dos objetivos. 
Ressalta-se, na Figura 2, que cada componente do Modelo é um nó ou subnó identificado pelo ícone em formato de esfera próximo ao rótulo do componente. Evidencia-se que as categorias não são exclusivas de um único artigo, pois um mesmo estudo pode informar nos seus resultados um ou mais componentes.

Após a codificação das fontes realizou-se a análise de cluster para visualizar os dados (etapa 3), por meio da identificação dos grupos de subnós. Para tanto, utilizou-se a técnica de análise de cluster por valor atribuído do software NVIVO 10 com coeficiente Jaccard que agrupa os elementos por similaridade. Este coeficiente considera dados binários, no qual se entende que: $1=$ presença; 0 =ausência. Neste caso específico foram inseridas características categóricas (atributos) que serviram de elementos comuns entre determinados nós, formando clusters. Quando os nós ou subnós recebem atributos, os mesmos passam a ser denominados de Cases, sendo assim cada categoria dos resultados um Case. Foram criados sete atributos para qualificar os Cases no NVIVO 10 (Quadro 2).

\begin{tabular}{|c|c|c|c|}
\hline \multicolumn{4}{|l|}{ Classificação de nós } \\
\hline Cases & Criado em 23/02/2015 13:24 & Criado por MR & \\
\hline Nome & Tipo & Criado em & \\
\hline Aspectos da criança & Texto & $23 / 02 / 2015$ & $13: 27$ \\
\hline Aspectos do contexto & Texto & $23 / 02 / 2015$ & $13: 26$ \\
\hline Aspectos da escola & Texto & $23 / 02 / 2015$ & $13: 26$ \\
\hline Aspectos da família & Texto & $23 / 02 / 2015$ & $13: 26$ \\
\hline Aspectos do transtorno & Texto & $23 / 02 / 2015$ & $13: 27$ \\
\hline Aspectos metodológicos & Texto & $23 / 02 / 2015$ & $13: 27$ \\
\hline Prevenção, intervenções e tratamento & Texto & $23 / 02 / 2015$ & $13: 27$ \\
\hline
\end{tabular}

Quadro 2. Atributos criados para qualificar os Cases.

No Quadro 2 observam-se os atributos criados para caracterizar categoricamente os Cases. Após a construção dos atributos procurou-se associá-los aos Cases envolvidos no estudo para posterior realização de análise de cluster por valor atribuído. Sendo assim, os Cases foram agrupados considerando as suas associações com os atributos supracitados (Figura 3).

A análise de cluster por valor atribuído aplicada aos Cases representativos das variáveis exploradas pelos artigos investigados resultou em sete categorias analíticas, a saber: (1) aspectos da família; (2) aspectos do transtorno; (3) aspectos metodológicos; (4) prevenção; (5) aspectos relativos do contexto; (6) aspectos relativos à escola; (7) aspectos relativos à criança. 


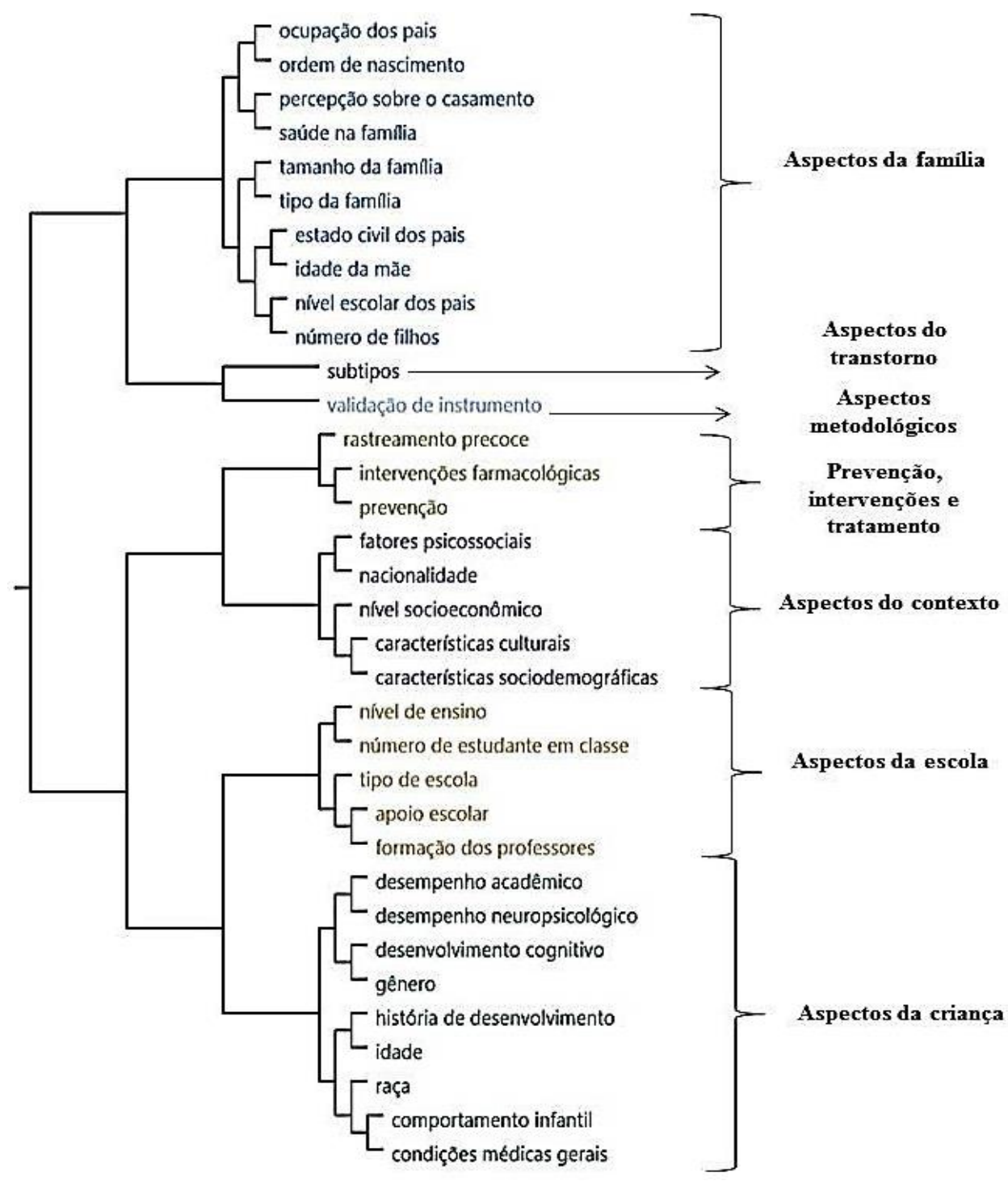

Figura 3. Dendograma: Agrupamentos dos Cases por Atributo

\section{Aspectos da Família}

Este grupo reuniu as seguintes categorias: ocupação dos pais; ordem de nascimento; percepção sobre casamento; saúde na família; tamanho da família; tipo de família; estado civil dos pais; idade da mãe; nível escolar dos pais; número de filhos.

A ocupação dos pais foi verificada em quatro artigos em diferentes países, a saber: Índia (Ajinkya et al., 2012), no Qatar (Bener et al., 2008, 2006) e, na República Democrática do Congo (Kashala et al., 2005) que analisaram as famílias cujas mães eram donas de casa e os pais, os que garantiam o sustento financeiro do lar, no entanto, não encontraram relação significativa com a estimativa de prevalência. Este dado foi confirmado na investigação realizada no Irão (Meysamie et al., 2011).

A variável ordem de nascimento foi investigada no Irão com 1658 crianças por Amiri et al. (2010) e na Nigéria com uma amostra de 200 crianças por Ndukuba et al. (2014). Nos dois estudos não foram identificadas diferenças significativas no diagnóstico do TDAH. Além desta variável, Ndukuba et al. (2014) relacionaram a percepção dos pais sobre o casamento e o TDAH e constataram uma relação significativa ao verificar que $14.3 \%$ das crianças cujos pais tinham uma percepção negativa do casamento possuíam o TDAH. Estes dados foram confirmados por Bener et al. (2008) e Bener et al. (2006) que também investigaram a avaliação dos pais em relação ao matrimônio. Por sua vez, a variável estado civil dos pais foi avaliada em um artigo (Ndukuba et al., 2014) que não sinalizou relação significativa com o transtorno.

Dois artigos (Jin et al., 2013; Ndukuba et al., 2014) investigaram a saúde da família, em particular, a saúde da mãe no período gestacional e a saúde do bebê ao nascer, todavia não encontraram relação significativa com o transtorno. Já um estudo (Meysamie et al., 2011) realizado no Irão com 160 mães que 
engravidaram entre os 18 e 35 anos, investigou a variável idade materna e o TDAH, mas não encontrou relação significativa.

Dois artigos analisaram as variáveis: tamanho da família e o número de filhos, a saber: Ndukuba et al. (2014) avaliou as famílias com menos ou mais de quatro filhos, e constatou uma relação significativa entre o diagnóstico do TDAH e as famílias com mais de quatro filhos, como também investigou os tipos de família (monogâmicas, poligâmicas e/ou monoparentais), entretanto não encontrou relação significativa. Este resultado foi confirmado no estudo (Ajinkya et al., 2012) que verificou os tipos de família diferenciando-as em nuclear, conjunta e família de três gerações.

Um estudo realizado em Xangai (Jin et al., 2013) analisou 5.453 questionários respondidos por pais e constatou uma maior prevalência do transtorno em crianças cujos pais tinham o nível educacional inferior ao segundo grau. Tal resultado foi confirmado nos EUA por Wolraich et al. (2012). No entanto, este dado não foi confirmado em cinco estudos (Ajinkya et al., 2012; Bener et al., 2008, 2006; Ndukuba et al., 2014) que relacionaram o diagnóstico do transtorno com a escolaridade dos pais.

\section{Aspectos do Transtorno}

Esta categoria é constituída apenas pela variável subtipos do TDAH. Compreende-se que os subtipos são: predominantemente desatento; predominantemente hiperativo/impulsivo ou o tipo combinado. Cinco artigos (Freire \& Pondé, 2005; Sánchez et al., 2014; Sánchez et al., 2011; Talaei et al., 2010; Vasconcelos et al., 2003) não encontraram diferenças significativas entre os três subtipos do TDAH. No entanto, um estudo, (Alloway et al., 2010) que investigou 964 meninos de 10 anos identificou maior frequência do subtipo hiperativo/impulsivo. Já Ndukuba et al. (2014) e Amiri et al. (2010) identificaram maior frequência do subtipo combinado. Enquanto que Ajinkya et al. (2011) e Jin et al. (2013) identificaram maior frequência do subtipo desatento.

Um estudo conduzido na Noruega (Ullebø et al., 2012) comparando os questionários dos pais e dos professores em relação aos subtipos do TDAH encontrou maior frequência do subtipo desatento segundo os pais. Em oposição, em três estudos, sendo um realizado na Colômbia (Cornejo et al., 2005); um na Arábia Saudita (Alqahtani, 2010) e outro na Espanha (Cardo et al., 2007) identificaram maior porcentagem do subtipo desatento no relato dos professores.

\section{Aspecto Metodológico}

Esta categoria é constituída apenas por um estudo. Abdekhodaie et al., (2012) realizaram no Irão uma pesquisa que se propôs comparar as pontuações obtidas através da entrevista clínica pautada no DSM-IV e, o Questionário de Conners, um instrumento composto de dez questões distribuídas em quatro escalas: atividade, concentração, hiperatividade e impulsividade. Os autores analisaram as respostas dos professores e dos pais de 1083 crianças em idade pré-escolar. Os resultados apontaram que o questionário é um adequado screening do TDAH. Embora, esta categoria abarque apenas um artigo, destacou-se a comparação de dois métodos de avaliação em uma mesma pesquisa com duas fontes de informação oriundos de dois contextos (familiar e escolar).

\section{Prevenção, Intervenções e Tratamento}

Este grupo é formado pelas variáveis: rastreamento precoce; intervenções farmacológicas; e prevenção. Tendo por objetivo verificar o rastreamento precoce, a pesquisa de Ndukuna et al. (2014) verificou que nenhuma das crianças identificadas com TDAH teve acesso ao sistema de saúde da zona rural do sudeste da Nigéria. Este dado revela o escasso sistema público de saúde neste país e a falta de acesso a medidas preventivas e de rastreamento precoce.

Seis estudos (Abdekhodaie et al., 2012; Alloway et al., 2010; Bener et al., 2006; Freire \& Pondé, 2005; Meysamie et al., 2011; Soma et al., 2009) ressaltam que o rastreamento precoce do TDAH é essencial para a elaboração de medidas de prevenção em relação ao desenvolvimento dessas crianças ao longo da vida.

Um artigo investigou a variável intervenção farmacêutica. Com uma amostra de 10427 crianças nos EUA, Wolraich et al. (2012) encontraram relação significativa com o diagnóstico do TDAH em crianças que recebiam tratamento medicamentoso.

\section{Aspectos Relativos do Contexto}

Constituído pelas seguintes variáveis: fatores psicossociais; nacionalidade; nível socioeconômico; características culturais e características sociodemográficas.

Os fatores psicossociais foram investigados em alguns artigos (Bener et al., 2008, 2006; Ndukuba et al., 2014) que avaliaram a relação afetiva dos pais e encontraram diferença significativa em relação a manifestação dos sintomas do TDAH nos filhos. Por sua vez, os estudos (Ajinkya et al., 2012; Bener et al., 
2008, 2006; Kashala et al., 2005) investigaram a ocupação dos pais, mas não encontraram relação significativa com o TDAH.

Em relação à nacionalidade, destacam-se dois estudos: uma pesquisa realizada em Xangai (Jin et al., 2013) que encontrou maior estimativa de prevalência do TDAH nas crianças estrangeiras, uma vez comparadas as crianças chinesas residentes no Distrito de Zhabei, e uma investigação realizada no Qatar que também apontou taxa de prevalência maior nos estrangeiros que nos cataris (Bener et al., 2008).

Sete estudos investigaram a variável socioeconômica (Ajinkya et al., 2012; Bener et al., 2008, 2006; Jin et al., 2013; Meysamie et al., 2011; Montiel-Nava et al., 2003; Wolraich et al., 2012) e encontraram relação significativa em relação ao TDAH. Por exemplo, Jin et al. (2013) verificaram a situação socioeconômica dos participantes em Xangai e constataram que as famílias que apresentavam uma renda mensal inferior a 1.000 yuan, manifestavam maior propensão ao transtorno. Wolraich et al. (2012) investigaram dois estados dos EUA, Oklahoma e Carolina do Sul, e encontraram relação significativa da presença do TDAH em crianças cujas famílias apresentavam renda anual inferior a 45000 US\$.

Seis artigos analisaram as características sociodemográficas através de questionários a fim de obterem informações correspondentes às famílias e ao desenvolvimento da criança. Duas investigaram a idade da mãe no período gestacional, mas não encontraram relação significativa (Jin et al., 2013; Ndukuba et al., 2014). Duas encontraram relação significativa com o diagnóstico do transtorno no que tange a situação socioeconômica da família, e outras duas verificaram o tipo de família e não encontraram diferença significativa (Ajinkya et al., 2012; Ndukuba et al., 2014).

\section{Aspectos Relativos à Escola}

Constituído pelas seguintes variáveis: nível de ensino; número de estudantes em classe; tipo de escola; apoio escolar; formação dos professores.

Dois estudos realizados no Irão, Amiri et al. (2010) e Talaei et al. (2010), avaliaram o nível de ensino e o diagnóstico do TDAH, mas não encontraram relação significativa. No que tange a variável número de estudantes em classe, um artigo (Talaei et al., 2010) não constatou correlação significativa.

No que concerne ao tipo de escola onde ocorreu a seleção das amostras, sete estudos utilizaram amostras de escolas públicas (Alloway et al., 2010; Bener et al., 2008, 2006; Fontana et al., 2007; MontielNava et al., 2003; Sánchez et al., 2011; Vasconcelos et al., 2003), ao passo que três (Amiri et al., 2010; Freire \& Pondé, 2005; Vasconcelos et al., 2003), foram realizados em escolas públicas e privadas.

Partindo da premissa de que os sintomas do TDAH podem comprometer o desempenho acadêmico, um estudo verificou a variável apoio escolar como forma de suporte às dificuldades escolares dos alunos em 12 escolas regulares do Reino Unido (Alloway et al., 2010), e constatou que os alunos com o transtorno são os mais demandantes de apoio. Já no que tange à variável formação dos professores em comparação com formação dos pais, também respondentes dos questionários de TDAH, um estudo realizado na Arábia Saudita (Alqahtani, 2010) não encontrou diferença significativa.

\section{Aspectos Relativos à Criança}

Este grupo é formado pelas seguintes variáveis: desempenho acadêmico, desempenho neuropsicológico, desenvolvimento cognitivo, gênero, história de desenvolvimento, idade, etnia, comportamento infantil e condições médicas gerais.

Em relação à variável desempenho acadêmico, um estudo (Bener et al., 2006) avaliou a performance acadêmica das crianças com e sem os sintomas do TDAH. Os autores constataram que aquelas que manifestavam os sintomas, sinalizaram desempenho acadêmico inferior aos seus colegas de classe sem o diagnóstico do TDAH. Já os estudos realizados na Colômbia (Cornejo et al., 2005) e no Panamá (Sánchez et al., 2011) sugerem maior repetição de ano por alunos que manifestam o transtorno.

Um estudo (Montiel-Nava et al., 2003) avaliou o desempenho neuropsicológico e o desenvolvimento cognitivo de crianças diagnosticadas com TDAH através de testes de inteligência WISCIII e WPPSI-R. Os seus resultados evidenciam que os estudantes classificados dentro do subtipo desatento apresentam um menor QI comparado aos demais subtipos. Por outro lado, a pesquisa realizada por Cornejo et al. (2005) que utilizou o WISC-R e o WAIS para avaliar a cognição das crianças e adolescentes, encontrou os menores QIs entre os participantes classificados dentro do subtipo hiperativo/impulsivo.

No que concerne ao comportamento infantil, um estudo (Bener et al., 2008) avaliou os comportamentos de desobediência, irritabilidade, condutas antissociais, e observou maior frequência nas crianças com TDAH, uma vez comparada ao grupo controle.

Em relação ao gênero, quatro estudos não encontraram diferença significativa entre o gênero feminino e masculino (Kashala et al., 2005; Ndukuba et al., 2014; Sánchez et al., 2011; Vasconcelos et al., 2003). Por sua vez, 14 estudos (Abdekhodaie et al., 2012; Alqahtani, 2010; Amiri et al., 2010; Bener et al., 2008, 2006; Cornejo et al., 2005; Fontana et al., 2007; Freire \& Pondé, 2005; Jin et al., 2013; Meysamie et 
al., 2011; Montiel-Nava et al., 2003; Sánchez et al., 2014; Soma et al., 2009; Ullebø et al., 2012) apontaram maior prevalência no sexo masculino.

No que tange a história do desenvolvimento, Sánchez et al. (2011) utilizaram o BASC-2 (Behavior Assessment System for Children, Second Edition) a fim de obter uma visão geral do desenvolvimento da criança, história clínica e desempenho acadêmico, comparando dois grupos: com e sem TDAH. Os dados indicaram diferenças entre os grupos, em que os pais com os filhos com TDAH utilizaram com mais frequência os serviços médicos e psicológicos em comparação com os que não manifestavam os sintomas do transtorno. Por sua vez, um estudo (Kashala et al., 2005) investigou a idade dos pais no período da gravidez e alguns fatores perinatais como a gravidez, o peso do bebê ao nascer e história médica, no entanto, não encontrou diferença significativa.

No que concerne à variável idade, um estudo realizado nos EUA (Wolraich et al., 2012) investigou os grupos de 5 a 13 anos e encontrou maior frequência do TDAH entre os participantes que se encontravam na faixa etária dos 8 aos 10 anos. Já o estudo realizado no Qatar (Bener et al., 2006) encontrou maior prevalência entre os dos 10 aos 12 anos. A investigação em Xangai (Jin et al., 2013) identificou maior frequência entre os de 7 a 10 anos. 0 estudo conduzido no Japão (Soma et al., 2009) sinalizou maior porcentagem dos 4 aos 5 anos. Não obstante, 5 estudos (Amiri et al., 2010; Bener et al., 2006; Cornejo et al., 2005; Kashala et al., 2005; Ndukuba et al., 2014) não identificaram relações entre a idade e o transtorno.

No tocante à etnia, três estudos (Fontana et al., 2007; Jin et al., 2013; Wolraich et al., 2012) não encontraram associação com a presença do transtorno. Por sua vez, quanto à avaliação das condições médicas como exploração complementária, um estudo realizado no Brasil (Vasconcelos et al., 2003) em uma escola pública primária, além de verificar os sintomas do TDAH, avaliou as possíveis anormalidades associadas, como: asma brônquica, distúrbio de ansiedade, distúrbios do sono, epilepsia parcial complexa, surdez e pediculose, a fim de estabelecer uma anamnese clínica mais acurada, contudo não analisou a relação destas patologias com o TDAH.

\section{DISCUSSÃO}

O foco desta revisão foi caracterizar através de um mapeamento de dados, 23 estudos de prevalência do TDAH realizados em diferentes áreas geográficas de quatro continentes (América, Ásia, Europa e África) objetivando construir um panorama destas investigações. Em relação à prevalência do transtorno, observou-se a média de $11.26 \%$ entre as estimativas de prevalência encontradas (95\% IC=8.4 -14.1; $p<$ 0.001), sendo que as mesmas variaram de $2.7 \%$ a $31.1 \%$ nos diferentes países. Em vista dessa discrepância, alguns dos estudos levantados (Alloway et al., 2010; Amiri et al., 2010; Cardo et al., 2007; Fontana et al., 2007; Kashala et al., 2005; Meysamie et al., 2011; Wolraich et al., 2012) apontaram que as estimativas encontradas não podem ser generalizadas a outros contextos geográficos.

Convém ressaltar que as maiores estimativas de prevalência foram encontradas em amostras de pré-escolares de 3 a 6 anos, apresentando médias de 25.8\% (Meysamie et al., 2011) e 31.1\% (Soma et al., 2009), ultrapassando a média percentual de 3\% a 7\% considerada pela Associação Psiquiátrica Americana (Willcutt, 2012), fato que põe em evidência os procedimentos metodológicos utilizados, a fonte de informação adotada como referência, os diferentes critérios diagnósticos aplicados (Cardo \& Servera, 2008; Faraone et al., 2003; Polanczyk, De Lima, Horta, Biederman, \& Rohde, 2007; Willcutt et al., 2012), o processo de seleção da amostra, e a idade dos sujeitos, já que as inquietações motoras tendem a ser mais evidenciadas na infância que na adolescência, o que pode resultar em subdiagnósticos ou hiperdiagnósticos (Russell a. Barkley et al., 2006; Catalá-López et al., 2012; Polanczyk et al., 2014).

Uma metanálise realizada por Wilcullt (2012) de 86 estudos envolvendo crianças e adolescentes, e 11 estudos com adultos, assinalou que a variação entre as estimativas de prevalência do transtorno podem ocorrer de acordo com os algoritmos de diagnóstico utilizados para definir o TDAH. Destacam que o controle dos mesmos, poderão minimizar essas divergências.

Em relação ao delineamento dos estudos levantados, observaram-se que a maioria, 99\%, foi do tipo transversal. Essa diferença acentuada pode ser em função das vantagens apresentadas no modelo transversal. Os estudos transversais (Ciappon, 2010) são eficientes nas investigações epidemiológicas por determinarem a prevalência em um dado momento, além de serem econômicos e não demandarem de um largo período de tempo, embora apresentem desvantagens como a possibilidade de omitirem alguns casos de prevalência, os vieses, e a falta de interpretação de causa-efeito, visto que, as informações dos fatores de risco e dos eventos ocorrerem de maneira simultânea.

De acordo com Fernández (2005), as vantagens dos estudos transversais estariam relacionadas com a representatividade da população e a elevada validade interna da amostra, enquanto que as desvantagens estariam relacionadas ao fator temporal que impossibilita a interpretação dos termos de causalidade, viabilizando apenas o estabelecimento de associações gerais. Contrastando as vantagens, 
Cardo e Severa (2008) destacam que os estudos transversais são limitados na identificação da prevalência do TDAH por não avaliarem as alterações neurocomportamentais que variam no desenvolvimento do indivíduo ao longo da vida, uma vez que se trata de um transtorno do desenvolvimento, estando vinculada a maturação do indivíduo.

Por outro lado, Fernández (2005) pontua que a vantagem de estudo epidemiológico do tipo longitudinal estaria relacionada ao evidente processo temporal de causa-efeito e à possibilidade de avaliar em múltiplas condições e exposições dos sintomas da patologia, embora apresente elevados custos financeiros. Dos 23 estudos categorizados, apenas uma investigação (Soma et al., 2009) apontou o desenho transversal como limitação metodológica. A quase unânime decisão dos autores na escolha do modelo transversal sugere que o fator temporal teve maior impacto no desenho do estudo.

Em relação ao processo de amostragem, os tipos diversificados sinalizaram os diferentes procedimentos utilizados pelos autores, fato que, supõem, o tamanho da população investigada, a disponibilidade dos participantes, e os ambientes de coleta de dados tenham influenciado na delimitação do tipo de seleção de amostra (Szwarcwald \& Damacena, 2008).

No tocante aos critérios diagnósticos utilizados pelos 23 artigos, observou-se que o DSM-IV foi um dos mais referenciados. 0 Manual Estatístico dos Transtornos Mentais, ainda que seja necessário para a classificação dos transtornos a nível global, é uma fonte de controvérsia entre os pesquisadores, tendo em vista que o diagnóstico se fundamentará exclusivamente na análise comportamental do indivíduo por não haver marcadores biológicos definidos (Cornejo et al., 2005), além de demandar experiência e conhecimento teórico por parte do profissional (Barbosa, 2001; Catalá-Lopez et al., 2012). Cabe ressaltar que nenhum dos artigos selecionados utilizou o CID-10 (Classificação Internacional de Doenças), sistema de classificação que apresenta diferentes critérios diagnósticos do TDAH em comparação com o DSM (Jara, 2009). Sugestiona-se que seria interessante a comparação dos dois sistemas de classificação em uma mesma amostra, por exemplo.

No que concerne às variáveis exploradas pelos pesquisadores, observaram-se divergências nos resultados publicados e como se tratam, na maioria, de estudos transversais, não se analisaram com precisão as relações de causa e efeito entre elas, a saber: a percepção negativa dos pais sobre casamento e o TDAH nos filhos, e o tamanho da família, possivelmente estariam relacionados à manifestação dos sintomas do transtorno que repercute diretamente na dinâmica familiar (Barkley, Guevremont, Anastopoulos, \& Fletcher, 1992; Jara, 2009; Polanczyk et al., 2014). Nas variáveis subtipos do transtorno, idade e gênero, observaram-se discordâncias entre os artigos, possivelmente esta divergência deve-se a diferentes tipos de amostras e critérios diagnósticos adotados (Catalá-López et al., 2012; Cordeiro et al., 2007; Polanczyk et al., 2014; Ramtekkar et al., 2010; Rohde et al., 2005; Willcutt et al., 2012).

Por sua vez, os fatores psicossociais, o nível socioeconômico familiar, desempenho acadêmico e cognitivo das crianças foram apontadas como tendo relação significativa com a sintomatologia do TDAH. Estes achados confirmam a necessidade da criação de medidas preventivas do transtorno (Alvarez \& Claros, 2012; Faraone et al., 2003; López-Soler, Puerto, López-Pina, \& Prieto, 2009; Polanczyk et al., 2014; Willcutt et al., 2012).

Com base nos dados apresentados, pode-se concluir que as diferentes estimativas de prevalência apresentadas variaram em função dos critérios metodológicos e diagnósticos adotados pelos pesquisadores, do processo de amostragem, como também, do contexto e dos instrumentos adotados na coleta dos dados (Catalá-López et al., 2012; Faraone et al., 2003; Polanczyk et al., 2007; Rohde et al., 2005; Willcutt et al., 2012).

\section{CONSIDERAÇÕES FINAIS}

O panorama característico dos estudos de prevalência do TDAH nos distintos países descritos neste estudo serve de base para outros pesquisadores interessados na temática em questão, pois ilustra os delineamentos dos estudos, os critérios metodológicos adotados, principalmente o uso do DSM como um consenso alargado na prática clínica, e as possíveis influências refletidas nas variadas estimativas de prevalência. Estas informações podem contribuir para a fundamentação de hipóteses de outros estudos.

É incontestável a necessidade de investigações com uma maior precisão metodológica para averiguar a magnitude das diversificadas estimativas de prevalência, uma vez que as variações das mesmas repercutirão diretamente na criação de políticas públicas voltadas a assistência a população com o transtorno.

Apesar das suas contribuições, o presente estudo apresenta limitações por considerar apenas uma base de dados no levantamento dos artigos, excluindo publicações em forma de dissertações, teses e capítulos de livros, como também, estudos não publicados apresentados em congressos. As prevalências das principais comorbilidades do TDAH também poderiam ter sido investigadas, o que ampliaria no quantitativo de estudos categorizados. 
Os resultados alcançados apoiam a premissa de que os critérios metodológicos e diagnósticos, a seleção das amostras, e a fonte de informação, ampliam a variabilidade das estimativas de prevalência a nível global. Futuras pesquisas poderão investigar a relação direta desses fatores no estabelecimento do diagnóstico do TDAH, e consequentemente na taxa de prevalência alcançada.

\section{Referências}

Abdekhodaie, Z., Tabatabaei, S. M., \& Gholizadeh, M. (2012). The investigation of ADHD prevalence in kindergarten children in northeast Iran and a determination of the criterion validity of Conners' questionnaire via clinical interview. Research in Developmental Disabilities, 33(2), 357-361. doi:10.1016/j.ridd.2011.10.006

Ajinkya, S., Kaur, D., Gursale, A., \& Jadhav, P. (2012). Prevalence of Parent-Rated Attention Deficit Hyperactivity Disorder and Associated Parent-Related Factors in Primary School Children of Navi Mumbai-A School Based Study. The Indian Journal of Pediatrics, 80(March), 207-210. doi:10.1007/s12098-012-0854-1

Alloway, T., Elliott, J., \& Holmes, J. (2010). The prevalence of ADHD-like symptoms in a community sample. Journal of Attention Disorders, 14(1), 52-56. doi:10.1177/1087054709356197

Alqahtani, M. M. J. (2010). Attention-deficit hyperactive disorder in school-aged children in Saudi Arabia. European Journal of Pediatrics, 169(9), 1113-1117. doi:10.1007/s00431-010-1190-y

Alvarez, C. V., \& Claros, J. a V. (2012). Trastorno por Deficit de Atencion e Hiperactividad (TDAH), una problematica a abordar en la política pública de primera infancia en Colombia. Revista de Salud Publica, 14(2), 113-128. doi:10.1590/S0124-00642012000800010

American Psychiatric Association. (2013). Highlights of Changes from DSM-IV-TR to DSM-5. American Psychiatric Association, Washington. doi:10.1176/appi.focus.11.4.525

Amiri, S., Fakhari, A., Maheri, M., \& Mohammadpoor Asl, A. (2010). Attention deficit/hyperactivity disorder in primary school children of Tabriz, North-West Iran. Paediatric and Perinatal Epidemiology, 24(6), 597-601. doi:10.1111/j.1365-3016.2010.01145.x

Barkley, R. a, Guevremont, D. C., Anastopoulos, a D., \& Fletcher, K. E. (1992). A comparison of three family therapy programs for treating family conflicts in adolescents with attention-deficit hyperactivity disorder. Journal of Consulting and Clinical Psychology, 60(3), 450-462. doi:10.1037/0022006X.60.3.450

Barkley, R. a., \& Cox, D. (2007). A review of driving risks and impairments associated with attentiondeficit/hyperactivity disorder and the effects of stimulant medication on driving performance. Journal of Safety Research, 38(1), 113-128. doi:10.1016/j.jsr.2006.09.004

Barkley, R. a., Edwards, G., Laneri, M., Fletcher, K., Metevia, L., Catalá-López, F., ... Alda-Díez, J. a. (2006). Social and Emotional Impairment in Children and Adolescents with ADHD and the Impact on Quality of Life. Psychological Medicine, 3(2), 1436-1441. doi:10.1176/appi.focus.11.4.525

Bener, A., Al Qahtani, R., Teebi, A. S., \& Bessisso, M. (2008). The prevalence of attention deficit hyperactivity symptoms in schoolchildren in a highly consanguineous community. Medical Principles and Practice, 17(6), 440-446. doi:10.1159/000151564

Bener, A., Qahtani, R. Al, \& Abdelaal, I. (2006). The prevalence of ADHD among primary school children in an Arabian society. Journal of Attention Disorders, 10(1), 77-82. doi:10.1177/1087054705284500

Biederman, J. (2005). Attention-deficit/hyperactivity disorder: A selective overview. Biological Psychiatry, 57(11), 1215-1220. doi:10.1016/j.biopsych.2004.10.020

Biederman, J., Spencer, T., Lomedico, a., Day, H., Petty, C. R., \& Faraone, S. V. (2012). Deficient emotional self-regulation and pediatric attention deficit hyperactivity disorder: a family risk analysis. Psychological Medicine, 42, 639-646. doi:10.1017/S0033291711001644

Cardo, E., \& Servera, M. (2008). Trastorno por déficit de atención/hiperactividad: Estado de la cuestión y futuras líneas de investigación. Revista de Neurologia, 46(6), 365-372.

Cardo, E., Servera, M., \& Llovera, J. (2007). Estimación de la prevalencia del trastorno por déficit de atención e hiperactividad en la población normal de la isla de Mallorca. Revista de Neurología, $44(1), 10-14$.

Catalá-López, F., Peiró, S., Ridao, M., Sanfélix-Gimeno, G., Gènova-Maleras, R., \& Catalá, M. a. (2012). Prevalence of attention deficit hyperactivity disorder among children and adolescents in Spain: a systematic review and meta-analysis of epidemiological studies. BMC Psychiatry, 12(1), 168. doi:10.1186/1471-244X-12-168

Ciappon, A. (2010). Artículo Especial: Guía de lectura crítica de estudios observacionales en epidemiología ( primera parte ) Critical appraisal guide of observational studies in epidemiology ( first part ). Evidencia. Actualización En La Práctica Ambulatoria, 13(4), 135-140. Retrieved from www.foroaps.org/files/1340ad86707f80da800b750a285777ea.pdf 
Cordeiro, A. M., Oliveira, G. M. De, Rentería, J. M., \& Guimarães, C. A. (2007). Revisão sistemática: uma revisão narrativa. Revista Do Colégio Brasileiro de Cirurgiões, 34(6), 428-431. doi:10.1590/S010069912007000600012

Cornejo, J. W., Osío, O., Sánchez, Y., Carrizosa, J., Sánchez, G., Grisales, H., ... Holguín, J. (2005). Prevalencia del trastorno por déficit de atención-hiperactividad en niños y adolescentes colombianos. Revista De Neurología, 40(12), 716-722.

Díaz, A., Jiménez, J., Rodríguez, C., \& Afonso, M. (2013). Consideraciones de los estudios de prevalencia del trastorno por déficit de atención con o sin hiperactividad (TDAH). Revista de Psicología Y Educación, 8(2), 155-170.

Dye, C. (2013). Investigaciones para una cobertura sanitaria universal. Revista de Analisis Economico, 9(1), 127-150. doi:10.2105/AJPH.2013.301254

Emerich, D. R., da Rocha, M. M., Silvares, E. F. D. M., \& Gonçalves, J. D. P. (2012). Diferenças Quanto ao Gênero entre Escolares Brasileiros Avaliados pelo Inventário de Comportamentos para Crianças e Adolescentes (CBCL/6-18). Psico-Pucrs, 43, 380-387.

Faraone, S. V, Sergeant, J., Gillberg, C., \& Biederman, J. (2003). The worldwide prevalence of ADHD: is it an American condition? World Psychiatry: Official Journal of the World Psychiatric Association (WPA), 2(2), 104-113.

Fernández, E. (2005). Estudios epidemiológicos (STROBE). Medicina Clínica, 125, 43-48. doi:10.1016/S0025-7753(05)72209-0

Fontana, R. D. S., De Vasconcelos, M. M., Werner, J., De Góes, F. V., \& Liberal, E. F. (2007). Prevalência de TDAH em quatro escolas públicas brasileiras. Arquivos de Neuro-Psiquiatria, 65(1), 134-137. doi:10.1590/S0004-282X2007000100027

Freire, A. C., \& Pondé, M. (2005). Estudo piloto da prevalência do transtorno de déficit de atenção e hiperatividade entre crianças escolares na cidade do Salvador, Bahia, Brasil. Arquivos de NeuroPsiquiatria, 63(2 B), 474-478. doi:10.1590/S0004-282X2005000300020

Froehlich, T. E., Lanphear, B. P., Epstein, J. N., Barbaresi, W. J., Katusic, S. K., \& Kahn, R. S. (2007). Prevalence, recognition, and treatment of attention-deficit/hyperactivity disorder in a national sample of US children. Archives of Pediatrics \& Adolescent Medicine, 161(9), 857-864. doi:10.1001/archpedi.161.9.857

Galvão, C. M., Sawada, N. O., \& Trevizan, M. A. (2004). Revisão sistemática: Recursos que proporciona a incorporação nas evidências na prática da enfermagem. Revista Latino-Americana de Enfermagem Latino-Americana Enfermagem, 12(3), 549-556.

Guizzo, B. S., Krziminski, C. O., \& Oliveira, D. (2003). O software QSR NVivo 2.0 na análise qualitativa de dados: Ferramenta para a pesquisa em ciências humanas e da saúde. Revista Gaúcha de Enfermagem, 24(1), 53-60.

Hernández-Avila, M., Garrido-Latorre, F., \& López-Moreno, M. (2000). Diseño de estudios epidemiológicos. Salud Pública de México, 42(2), 144-154.

Hodgkins, P., Arnold, L. E., Shaw, M., Caci, H., Woods, J. K., \& Young, S. (2012). A systematic review of global publication trends regarding long-term outcomes of ADHD. Frontiers in Psychiatry, 2(JAN), 1-18. doi:10.3389/fpsyt.2011.00084

Jara Segura, A. B. (2009). El TDAH, Trastorno por Déficit de Atención con Hiperactividad, en las clasificaciones diagnósticas actuales ( C . I . E . 10 , D . S . M . IV - R y C . F . T . M . E . A .- R 2000). Norte de Salud Mental, 30-40.

Jin, W., Du, Y., Zhong, X., \& David, C. (2013). Prevalence and contributing factors to attention deficit hyperactivity disorder: A study of five- to fifteen-year-old children in Zhabei District, Shanghai. Asia-Pacific Psychiatry, 1-8. doi:10.1111/appy.12114

Kashala, E., Tylleskar, T., Elgen, I., Kayembe, K. T., \& Sommerfelt, K. (2005). Attention deficit and hyperactivity disorder among school children in Kinshasa, Democratic Republic of Congo. African Health Sciences, 5(3), 172-181.

Lage, M. (2011). Utilização do software NVivo em pesquisa qualitativa: uma experiência em EaD. $\begin{array}{llllll}\text { Educação Temática } & \text { Digital, } & 12, & 198-226 . & \text { Retrieved }\end{array}$ http://www.ssoar.info/ssoar/handle/document/24372

López-Soler, C., Puerto, J. C., López-Pina, J. A., \& Prieto, M. (2009). Percepción de los estilos educativos parentales e inadaptación en menores pediátricos. Anales de Psicologia, 25(1), 70-77.

López-Soler, C., Sáez, M. C., López, M. A., Fernández, V. F., \& Pina, J. a L. (2009). Prevalencia y características de los síntomas externalizantes en la infancia. Diferencias de género. Psicothema, $21,353-358$.

McGough, J. J., \& McCracken, J. T. (2006). Adult attention deficit hyperactivity disorder: Moving beyond DSM-IV. American Journal of Psychiatry, 163(10), 1673-1675. doi:10.1176/appi.ajp.163.10.1673 
Meysamie, A., Fard, M. D., \& Mohammadi, M. (2011). Prevalence of Attention-Deficit/Hyperactivity Disorder Symptoms in Preschool-aged Iranian Children. Iranian Journal of Pediatrics, 21(4), 46772. http://www.pubmedcentral.nih.gov/articlerender.fcgi?artid=3446126\&tool=pmcentrez\&renderty pe=abstract

Montiel-Nava, C., Peña, J. ., \& Montiel-Barbero, I. (2003). Datos epidemiológicos del trastorno por déficit de atención con hiperactividad en una muestra de niños marabinos, 37(9), 815-819.

Ndukuba, a. C., Odinka, P. C., Muomah, R. C., Obindo, J. T., \& Omigbodun, O. O. (2014). ADHD Among Rural Southeastern Nigerian Primary School Children: Prevalence and Psychosocial Factors. Journal of Attention Disorders. doi:10.1177/1087054714543367

Polanczyk, G., De Lima, M. S., Horta, B. L., Biederman, J., \& Rohde, L. A. (2007). The worldwide prevalence of ADHD: A systematic review and metaregression analysis. American Journal of Psychiatry, 164(6), 942-948. doi:10.1176/appi.ajp.164.6.942

Polanczyk, G., De Lima, M. S., Horta, B. L., Biederman, J., Rohde, L. A., Thapar, A., ... Young, J. (2014). Trastorno por déficit de atención e hiperactividad (TDAH) en adolescentes. Neurotherapeutics, 26(1), 422-433. doi:10.1016/j.rmclc.2015.02.005

Ramtekkar, U. P., Reiersen, A. M., Todorov, A. a, \& Todd, R. D. (2010). Sex and age differences in attentiondeficit/hyperactivity disorder symptoms and diagnoses: implications for DSM-V and ICD-11. Journal of the American Academy of Child and Adolescent Psychiatry, 49(3), 217-228.e1-e3. doi:10.1016/j.jaac.2009.11.011

Ranby, K. W., Boynton, M. H., Kollins, S. H., McClernon, F. J., Yang, C., \& Fuemmeler, B. F. (2012). Understanding the phenotypic structure of adult retrospective ADHD symptoms during childhood in the United States. Journal of Clinical Child and Adolescent Psychology: The Official Journal for the Society of Clinical Child and Adolescent Psychology, American Psychological Association, Division 53, 41(3), 261-74. doi:10.1080/15374416.2012.654465

Rohde, L. A., Szobot, C., Polanczyk, G., Schmitz, M., Martins, S., \& Tramontina, S. (2005). Attentiondeficit/hyperactivity disorder in a diverse culture: Do research and clinical findings support the notion of a cultural construct for the disorder? Biological Psychiatry, 57(11), 1436-1441. doi:10.1016/j.biopsych.2005.01.042

Sánchez, C., Ramos, C., \& Díaz, L. (2014). Attention-Deficit / Hyperactivity Disorder : prevalence of risk in the scholastic scope of the Canary islands, 42(4), 169-175.

Sánchez, E. Y., Velarde, S., \& Britton, G. B. (2011). Estimated prevalence of attention-deficit/hyperactivity disorder in a sample of Panamanian school-aged children. Child Psychiatry and Human Development, 42(2), 243-255. doi:10.1007/s10578-010-0213-2

Servera, M., \& Cardo, E. (2007). ADHD Rating Scale-IV en una muestra escolar española: Datos normativos y consistencia interna para maestros, padres y madres. Revista de Neurologia, 45(7), 393-399.

Soma, Y., Nakamura, K., Oyama, M., Tsuchiya, Y., \& Yamamoto, M. (2009). Prevalence of attentiondeficit/hyperactivity disorder (ADHD) symptoms in preschool children: Discrepancy between parent and teacher evaluations. Environmental Health and Preventive Medicine, 14(2), 150-154. doi:10.1007/s12199-008-0075-4

Szwarcwald, C. L., \& Damacena, G. N. (2008). Amostras complexas em inquéritos populacionais: planejamento e implicações na análise estatística dos dados. Revista Brasileira de Epidemiologia, 11(supl 1), 38-45. doi:10.1590/S1415-790X2008000500004

Talaei, A., Mokhber, N., Abdollahian, E., Bordbar, M. R. F., \& Salari, E. (2010). Attention deficit/hyperactivity disorder: a survey on prevalence rate among male subjects in elementary school (7 to 9 years old) in Iran. Journal of Attention Disorders, 13, 386-390. doi:10.1177/1087054708329886

Ullebø, A. K., Posserud, M. B., Heiervang, E., Obel, C., \& Gillberg, C. (2012). Prevalence of the ADHD phenotype in 7-to 9-year-old children: Effects of informant, gender and non-participation. Social Psychiatry and Psychiatric Epidemiology, 47, 763-769. doi:10.1007/s00127-011-0379-3

Vaquerizo-madrid, J. (2005). Hiperactividad en el niño preescolar: descripción clínica, 40(Supl 1), 25-32.

Vasconcelos, M. M., Werner, J., De Araújo Malheiros, a. F., Negreiros Lima, D. F., Oliveira Santos, Í.' S., \& Bardawil Barbosa, J. (2003). Prevalência do transtorno de déficit de atenção/hiperatividade numa escola pública primária. Arquivos de Neuro-Psiquiatria, 61(1), 67-73. doi:10.1590/S0004282X2003000100012

Willcutt, E. G. (2012). The Prevalence of DSM-IV Attention-Deficit/Hyperactivity Disorder: A MetaAnalytic Review. Neurotherapeutics, 9(3), 490-499. doi:10.1007/s13311-012-0135-8

Willcutt, E. G., Alvarez, C. V., Claros, J. a V., Barkley, R. A., Cardo, E., Servera, M., ... Steinau, S. (2012). The Prevalence of DSM-IV Attention-Deficit/Hyperactivity Disorder: A Meta-Analytic Review. Journal of 
Prevalência do TDAH

the American Academy of Child and Adolescent Psychiatry, 9(1), 52-58. doi:10.1097/01.CHI.0000026602.17655.e7

Wolraich, M. L., McKeown, R. E., Visser, S. N., Bard, D., Cuffe, S., Neas, B., ... Danielson, M. (2012). The Prevalence of ADHD: Its Diagnosis and Treatment in Four School Districts Across Two States. Journal of Attention Disorders. doi:10.1177/1087054712453169

$\begin{array}{ll}\text { Historial do } & \text { artigo } \\ \text { Recebido } & 30 / 04 / 2015 \\ \text { Aceite } & 25 / 10 / 2015 \\ \text { Publicado } & 12 / 2015\end{array}$

Apoio à publicação: Fundação para a Ciência e a Tecnologia (Ministério da Educação e Ciência, Portugal) - Programa FACC 